\title{
A new representation of acid-base disturbances
}

\author{
M. Hekking ${ }^{a}$, E.S. Gelsema*a, J. Lindemans ${ }^{b}$ \\ ${ }^{a}$ Department of Medical Informatics, Erasmus University, Postbus 1738, 3000 DR Rotterdam. The Netherlands \\ ${ }^{b}$ Laboratory for Clinical Chemistry, University Hospital Dijkzigt, Rotterdam. The Netherlands
}

(Received 9 June 1993; accepted 6 September 1993)

\begin{abstract}
The acid-base status of intensive care patients is monitored on the basis of three quantities. The graphical representation which may be of help for the monitoring task is therefore cumbersome. The classical Siggaard-Andersen acid-base chart is such a representation, but it is only suited for evaluating one acid-base status at a time and not for representing acid-base paths. A new representation, obtained after a principal components transformation is presented. It is shown that the representation is characteristic for the laboratory instrument used. Its most attractive feature is that it is distortionless with respect to the three-dimensional configuration.
\end{abstract}

Key words: Acid-base status; Blood chemistry; Intensive care; Principal components; Graphics

\section{Introduction}

The determination and use of multivariate reference ranges in clinical chemistry continues to attract attention. Two basic problems in the determination of reference ranges are (1) the selection of the population from which to obtain them, and (2) the selection of a statistical model. If a population of healthy persons is taken as a reference population, the reference ranges are often too narrow to be of practical use in clinical situations. Moreover, for quantities which are obtained by invasive procedures, the use of healthy volunteers may be ethically unsound. An alternative is to obtain values from a clinical population and estab-

\footnotetext{
* Corresponding author.
}

lish reference ranges on the basis of their statistical distribution. A drawback of such a procedure is that the ranges thus obtained are not universally valid and cannot be treated as standard reference ranges. The statistical model used for the determination of reference ranges is also of importance. Most reference ranges of batteries of chemical quantities are obtained using univariate techniques, even if it is known that (sometimes even strong) correlations exist. The use of reference ranges thus obtained leads to an overestimation of the percentage of abnormals. For instance, even for three uncorrelated tests, $86 \%(95 \%$ of $95 \%$ of $95 \%$ ) of the cases tested will fall in all of the three $95 \%$ normal reference ranges. So, using more than one univariate reference range, an overestimation of the percentage of abnormals can be expected 
per definition. These problems have been reconsidered for the variates reflecting the acid-base equilibrium in patients in an intensive care unit as study material. Both problems alluded to above occur in this situation: use of the standard, univariate reference ranges for the three correlated variates $\mathrm{pH}, \mathrm{PCO}_{2}$ and base excess (BE) in a population of intensive care patients has been shown to lead to fractions of abnormals in excess of $60 \%$ [1]. The use of the population in the clinic as its own reference requires a statistical technique to define 'normality'. For that purpose, a multivariate technique has been developed [1,2]. Use of reference regions based on such procedures yielded fractions of abnormals smaller than $25 \%$ [1]. This technique featured a multivariate distance, called the Mahalanobis distance. It was hypothesized that the distance between a standard reference point $\left(\mathrm{pH}=7.4, \mathrm{PCO}_{2}=40 \mathrm{mmHg}^{\mathrm{a}}\right.$ and $\left.\mathrm{BE}=0.0\right)$ and a point representing a patient, correlated with the severeness of the acid-base disturbance in that specific patient.

Although the use of clinic-dependent reference ranges may well serve to identify those patients most in need of care, preliminary studies in intensive care units showed that the acceptance was rather low, due to three reasons:

1. In some regions of the variate space, the Mahalanobis distance was extremely sensitive to small variations in the individual measurements.

2. There was a discrepancy in the classifications made by physicians and classifications made by the three-dimensional model.

3. A graphical representation of the variate space required three-dimensional graphical software with on-line rotation capabilities. This is no problem for demonstration purposes but cannot be expected to be used for the inspection of individual cases in a clinical setting.

This paper describes an alternative way of representation of the three variates, based on their

\footnotetext{
Although the kilopascal ( $\mathrm{kPa}$ ) recently replaced millimeters of mercury $(\mathrm{mmHg})$ as a unit of pressure, $\mathrm{mmHg}$ is still used in some hospitals. $1 \mathrm{mmHg}=0.1333 \mathrm{kPa}$
}

well-known interdependence. It will be shown that acid-base disturbances need not to be presented in three dimensions for an accurate evaluation of acid-base paths. Exploiting the fact that the problem is intrinsically almost two-dimensional (see also Madias et al. [3]), acid-base paths are represented without the distortion that is observed in the well-known Siggaard-Andersen diagram [4]. This is illustrated in Section 3. The possible use of such a chart for patient monitoring is discussed in Section 4 . Section 2 provides the rationale for the new chart derived from some basic principles of acid-base physiology.

\section{A new look at acid-base parameters}

The acid-base metabolism is monitored mainly on the basis of three parameters: $\mathrm{pH}, \mathrm{PCO}_{2}$ and base excess (BE) [5]. Although the use of $\mathrm{BE}$ is debated [6,7], it is used here, since BE is a widely used parameter in most European countries, including The Netherlands.

It is well known that the bicarbonate system is the most physiologically important buffering system in the human body. It can be denoted by:

$\mathrm{CO}_{2}+\mathrm{H}_{2} \mathrm{O} \Leftrightarrow \mathrm{H}_{2} \mathrm{CO}_{3} \Leftrightarrow \mathrm{H}^{+}+\mathrm{HCO}_{3}^{-}$

Applying the law of mass action to the right-hand side of this chemical reaction, the proton concentration $\left(\left[\mathrm{H}^{+}\right]\right)$can be expressed as a ratio of the carbonic acid concentration $\left(\left[\mathrm{H}_{2} \mathrm{CO}_{3}\right]\right)$ and the bicarbonate ion concentration $\left(\left[\mathrm{HCO}_{3}^{-}\right]\right)$, multiplied by a dissociation constant $K[8]$ :

$$
\left[\mathrm{H}^{+}\right]=K \frac{\left[\mathrm{H}_{2} \mathrm{CO}_{3}\right]}{\left[\mathrm{HCO}_{3}\right]}
$$

Taking the negative logarithm of each component in this formula and replacing $\left[\mathrm{H}_{2} \mathrm{CO}_{3}\right]$ by the concentration of dissolved $\mathrm{CO}_{2}$ (which may be written as the product of the solubility constant $\alpha$ and the partial pressure of $\mathrm{CO}_{2}$ in a blood sample) yields the well-known Henderson-Hasselbalch equation [9]:

$$
\mathrm{pH}=\mathrm{p} K^{\prime}+\log \frac{\left[\mathrm{HCO}_{3}^{-}\right]}{\alpha \mathrm{PCO}_{2}}
$$


with $\mathrm{p} K^{\prime}=6.10$ and $\alpha=0.225 \mathrm{mmol} / \mathrm{l} / \mathrm{kPa}$ (or $0.03 \mathrm{mmol} / \mathrm{l} / \mathrm{mmHg}$ ).

From this formula it can be seen that $\mathrm{pH}$, $\log \left[\mathrm{HCO}_{3}^{-}\right]$and $\log \mathrm{PCO}_{2}$ are linearly related.

To overcome a major drawback of $\left[\mathrm{HCO}_{3}^{-}\right]$as a metabolic indicator - $\left[\mathrm{HCO}_{3}^{-}\right]$is influenced by respiration - Astrup et al. defined the $\mathrm{BE}$ as 'the amount of acid (negative BE) or base (positive BE) required to titrate 1 liter of blood at a temperature of $38^{\circ} \mathrm{C}$ and a $\mathrm{PCO}_{2}$ of $40 \mathrm{mmHg}$ to a $\mathrm{pH}$ of $7.4^{\prime}$ [5]. Basically it is the difference between the $\left[\mathrm{HCO}_{3}^{-}\right]$of a blood sample at a $\mathrm{pH}$ of 7.4 and the normal reference value for $\left[\mathrm{HCO}_{3}^{-}\right]$, which is 24 $\mathrm{mmol} / \mathrm{l}$ [10]. Normal blood has a BE of 0 . BE can be calculated from measured $\mathrm{PCO}_{2}$ and $\mathrm{pH}$ with use of the Henderson-Hasselbalch equation and the hemoglobin concentration [10].

Because $\mathrm{BE}$ is a difference in concentrations, it is not possible to represent it in a logarithmic form. Therefore the strict linear dependence no longer holds when $\mathrm{BE}$ is substituted for $\left[\mathrm{HCO}_{3}^{-}\right]$ as a metabolic parameter in the diagnosing process. Use of $\mathrm{PCO}_{2}$ rather than $\log \mathrm{PCO}_{2}$ leads to another departure from strict linearity.

In the laboratory equipment, $\mathrm{pH}$ and $\mathrm{PCO}_{2}$ are measured and, based on these variables and the hemoglobin concentration, a calculated valuc of BE is issued. Different equipment implements different formulas for these calculations, e.g. measured or fixed values for hemoglobin concentrations may be used and corrections for temperature may or may not be applied. It is important to realize here that the intrinsic relationship between the values of $\mathrm{pH}$ and $\mathrm{PCO}_{2}$ on the one hand and $\mathrm{BE}$ on the other, is only dependent on the algorithm as implemented in the instrument used. The question as to the absolute value of the differences between instruments is addressed in another section below.

During earlier studies [1,2], large populations of acid-base variates were explored, using amongst others a multivariate statistical technique to estimate reference regions and graphical software with capabilities of on-line three-dimensional rotation. During these studies and the discussion of results with clinicians, it became clear that the correlation between the Mahalanobis distance (which may be interpreted as a severity index) and the severity assigned by the clinician based on all clinical information was only small. This may be attributed to the fact that the three-dimensional Mahalanobis distance is calculated using the inverse of an almost singular covariance matrix. Furthermore, ideally, a graphical representation, allowing the clinician to monitor the acid-base status over time, required the use of three-dimensional graphical software with options of on-line rotation. It is unlikely that a clinician will use such software in a clinical setting. During these evaluations. however, it was soon realized that, when plotting the combinations of the three variates as they occur in practice in three-dimensional space, the points are located on a surface with only a slight curvature. This surface will be called the realizable surface. Fig. 1 displays such a patient population in three dimensions. The cube in the middle is the volume in three dimensions which represents the standard normal reference volume as used in the Dijkzigt University Hospital Clinic (see Table 1). The surface of realizable points by definition contains the point $\mathrm{pH}=7.40, \mathrm{PCO}_{2}=40(\mathrm{mmHg}), \mathrm{BE}=0.0$ (according to the definition of the base excess). The intersection of the realizable surface with the cube is the realizable reference region. The standard definitions of acid-base disturbances (Table 1) describe regions in parameter space separated by the surface planes of the reference cube and their extensions. The intersections of the realizable surface with these regions are the realizable manifestations of the corresponding disturbances. In the following section, the calculation of the realizable surface from (large) collections of observations and the departure of this surface from linearity is described. The translation from the definitions of disturbances as contained in Table 1 to regions on the realizable surface is also considered.

\section{The principal components transformation}

Having observed that the relationship between $\mathrm{pH}, \mathrm{PCO}_{2}$ and $\mathrm{BE}$ is a nearly linear one, it is interesting to arrive at a mathematical description of this relationship and to investigate its departure from linearity. The mathematical technique to be used for such an investigation is the principal components transformation. In terms of linear algebra, for a set of $N$ vectors $\boldsymbol{x}^{k}(k=1, \ldots, N)$ in an 


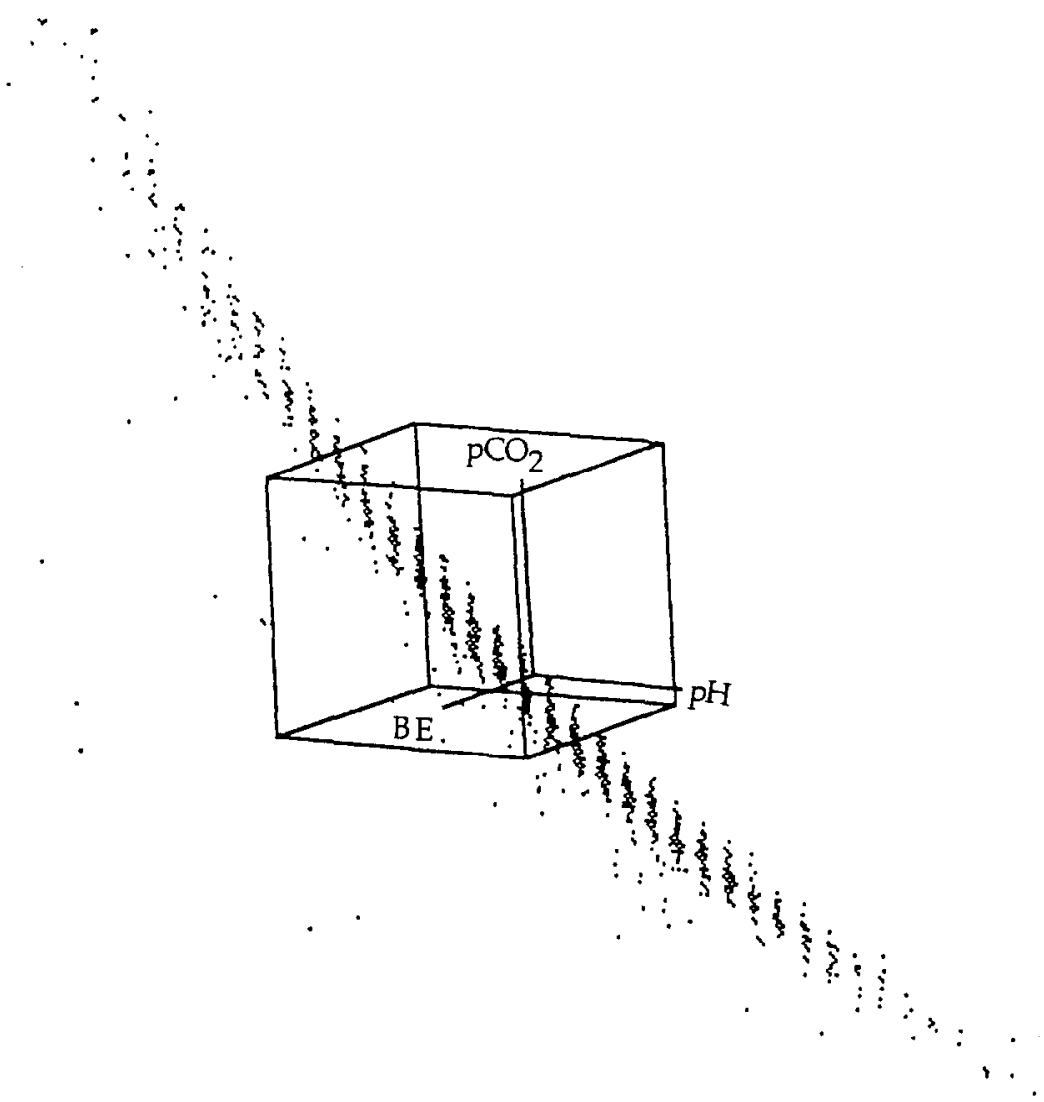

Fig. 1. A three-dimensional view of acid-base measurements. The direction of the three axes is indicated. The cube represents the standard normal reference area as defined in Table 1 . The points are concentrated on a surface with a slight curvature.

Table 1

The classical definitions of the various acid-base conditions

\begin{tabular}{llll}
\hline State & $\mathrm{pH}$ & $\mathrm{PCO}_{2}$ & $\mathrm{BE}$ \\
\hline Normal & 7.35 to 7.45 & 4.70 to 6.40 & -3.0 to 3.0 \\
Uncompensated respiratory acidosis & - & + & 0 \\
Partly compensated respiratory acidosis & - & + & + \\
Fully compensated respiratory acidosis & 0 & + & + \\
Partly compensated metabolic alkalosis & + & + & + \\
Uncompensated metabolic alkalosis & + & - & + \\
Metabolic and respiratory alkalosis & + & - & 0 \\
Uncompensated respiratory alkalosis & + & - & - \\
Partly compensated respiratory alkalosis & + & - & - \\
Fully compensated respiratory alkalosis & 0 & - & - \\
Partly compensated metabolic acidosis & - & 0 & - \\
Uncompensated metabolic acidosis & - & + & -
\end{tabular}

The normal condition is given in terms of the standard reference ranges. For each variate, the standard reference range is given as the range between mean value +2 S.D. (standard deviation) and mean value -2 S.D. Therefore, S.D. values for $\mathrm{pH}, \mathrm{PCO} 2$ and $\mathrm{BE}$ are $0.025,0.425$ and 1.5, respectively. $\mathrm{PCO}_{2}$ is expressed in $\mathbf{k P a}, \mathrm{BE}$ is expressed in $\mathrm{mmol} / \mathrm{l}$. A condition is defincd to be normal if all three measurements fall inside the standard reference range. For the abnormal conditions, the variates are given relative to the standard normal reference ranges. Below, inside and above the reference range is indicated by,- 0 and + , respectively. 
n-dimensional space, the variance-covariance matrix $V$ is defined by:

$$
V=\frac{\sum_{k=1}^{N}\left(x^{k}-m\right)\left(x^{k}-m\right)^{\mathrm{T}}}{N(N-1)}
$$

where $\boldsymbol{m}$ is the vector of the mean of the set $\boldsymbol{x}^{k}$ $(k=1, \ldots, N)$ and the superscript $\mathrm{T}$ indicates transposition of a vector, in the convention that an untransposed vector is a column vector.

A classical problem in linear algebra is to find a matrix of transformation $U$, which transforms the original vectors $\boldsymbol{x}$ into vectors $\boldsymbol{y}$, according to $y=U x$, such that the variance covariance matrix $W=U V U^{\mathbf{T}}$ of the transformed vectors $y$ is a diagonal matrix. This problem is known as an eigenvalue problem and the resulting transformation is the eigenvector transformation or principal component transformation. If $U$ is constrained to be a unitary matrix, the component variances of the transformed vectors $y$ appear as the eigenvalues in the analysis.

Since the eigenvector transformation diagonalizes the variance-covariance matrix, the variance in the set of vectors on which the transformation is defined is decomposed into $n$ orthogonal directions. Thus, for a set of $n$-dimensional vectors for which it is observed that (most of) the variance is confined to a subspace of dimension $p<n$, it is expected that the components 1 through $p$ of the transformed vectors contain (most of) the useful information. The components $p+1$ through $n$ have only a small variance, and thus convey (almost) no information.

In the present application, $n=3$ and due to the linear relationship indicated in Section 2 it is expected that $p=2$. The principal components transformation was calculated as follows: a sample of 1500 measurements of $\mathrm{pH}, \mathrm{PCO}_{2}$ and $\mathrm{BE}$ was first standardized:

$z_{i}=\frac{x_{i}-x 0_{i}}{s_{i}}$

where $x 0_{i}$ is $7.40,40.00(\mathrm{mmHg})$, and 0.00 , for $i=1,2$ and 3 , respectively, and $s_{i}$ is the corresponding standard deviation as listed in Table 1. The $z_{i}$ values are therefore the deviations from the
Table 2

The three eigenvalues $(\lambda)$ and corresponding eigenvectors $(e)$ of the principal component transformation as obtained from the first data set of 1500 patient measurements

\begin{tabular}{llll}
\hline$i$ & 1 & 2 & 3 \\
\hline$\lambda_{i}$ & 13.635 & 7.769 & 0.073 \\
$e_{i}$ & $(0.49,0.22,0.84)$ & $(-0.59,0.80,0.14)$ & $(0.64,0.75,-0.52)$ \\
\hline
\end{tabular}

point $x 0$ measured in units of the corresponding standard deviations.

The eigenvalues $\lambda$ and eigenvectors $e$ of the variance-covariance matrix of the set of the $1500 z$ vectors were then determined. The result is listed in Table 2.

It is apparent that, indeed, the variance in the third principal component is extremely small as

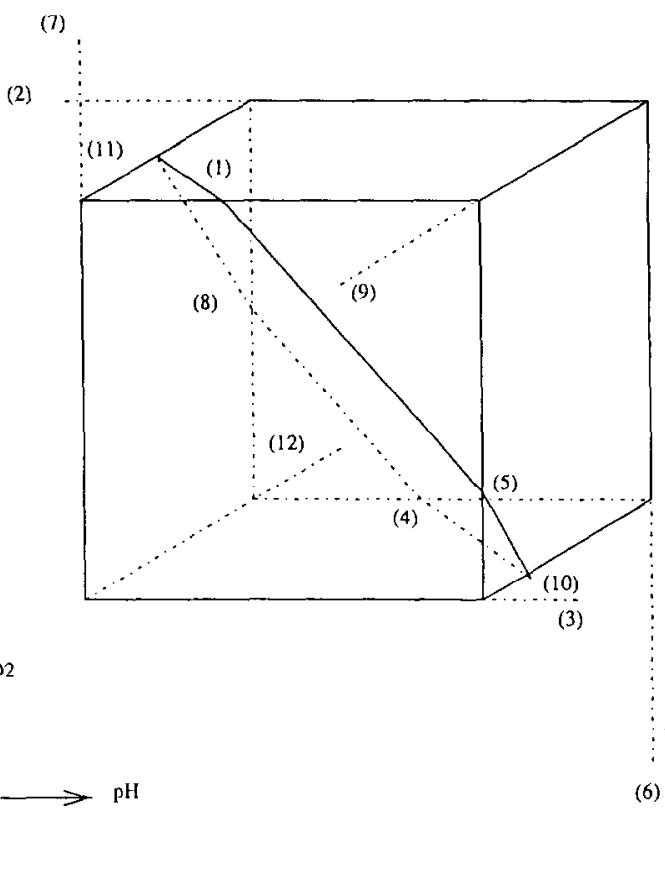

Fig. 2. The standard normal reference cube and the plane spanned by the first two principal components, as determined from a sample of 1500 acid-base measurements. The points of intersection of the plane with the ribs of the cube (or their extensions) are numbered I through 12. 
compared with the total variance. The components 1,2 and 3 explain $63.5 \%, 36.2 \%$ and $0.3 \%$ of the total variance, respectively. The plane spanned by the first two eigenvectors is the realizable plane. The proposed chart is the projection onto this plane. In order to give the chart a standard appearance, an additional rotation in the realizable plane is added such that the positive projected $\mathrm{pH}$ direction points to the right.

Fig. 2 illustrates the situation. The cube

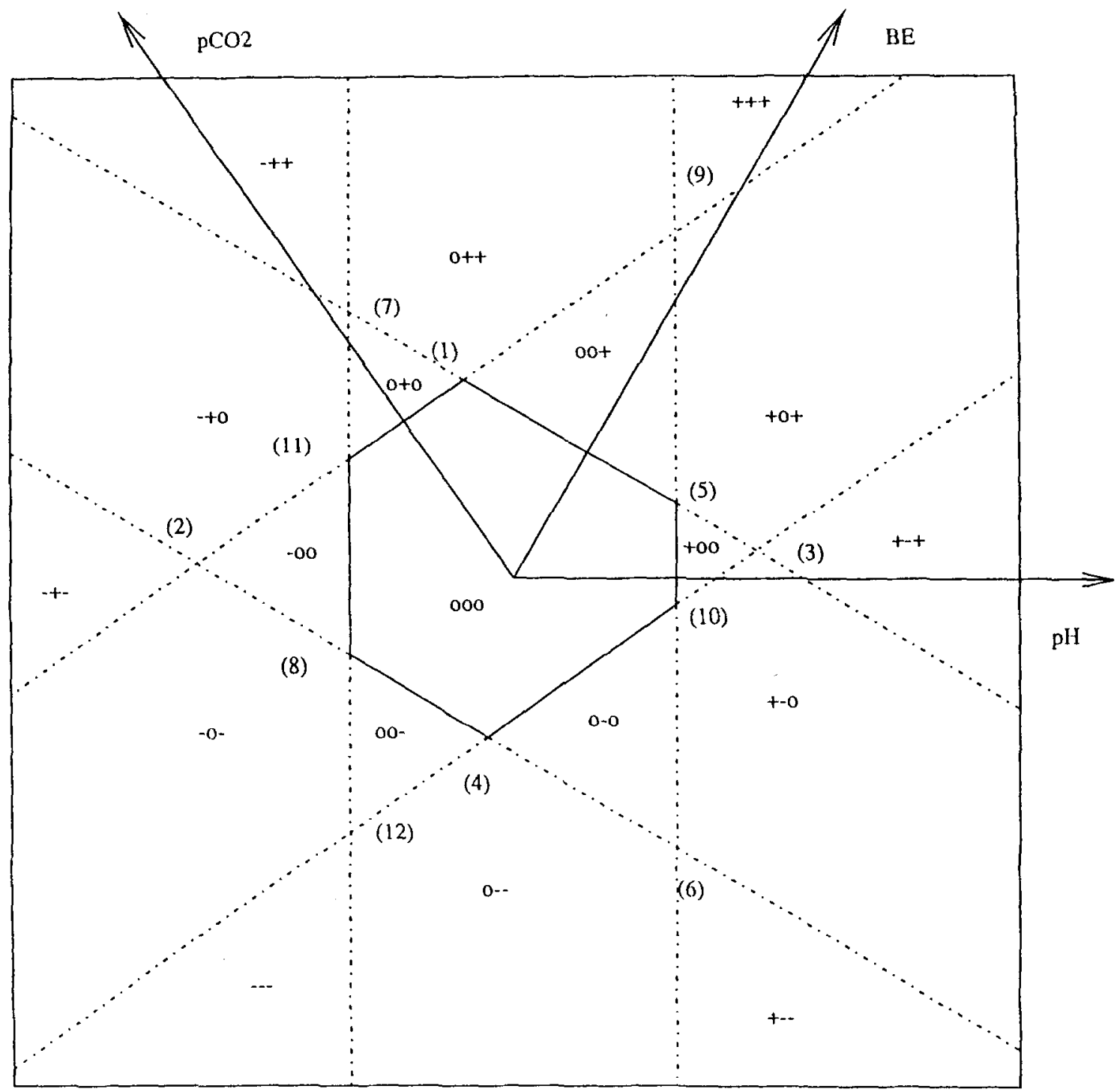

Fig. 3. The plane spanned by the two first principal components. The arrows are the projections of the positive directions of pH, $\mathrm{PCO}_{2}$ and $\mathrm{BE}$ on the plane. The points of intersection with the standard reference cube (Fig. 2) are indicated with numbers (I through 12). The areas corresponding to the various acid-base disturbances (Table 1) are indicated with a three character code (e.g. +-+ ). The central hexagon is the representation of the standard normal reference region. 
represents the standard reference volume according to the rules in Table 1. For all points inside the cube, each of the variates is within its univariate standard reference range. The plane intersecting the cube is the plane spanned by the first two prin- cipal components. Since $99.7 \%$ of the total variance is concentrated in this plane, projection of the original points on this plane is almost distortionless. In the terms of Section 2, the realizable surface can very well be approximated by a

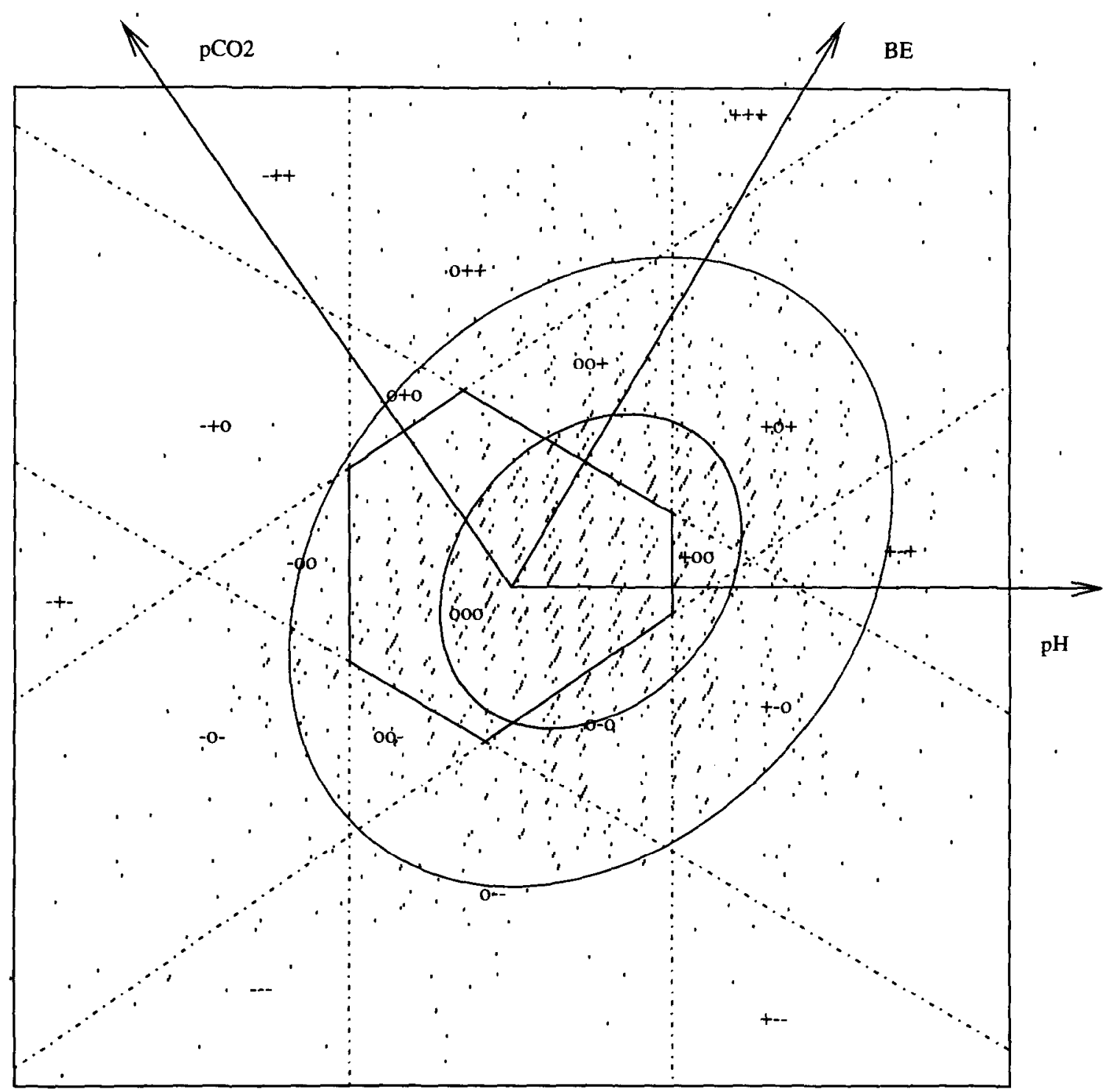

Fig. 4. The new acid-base chart with points corresponding to 1500 acid-base measurements. These measurements were used to calculate the coordinates of the principal component plane. A Gaussian distribution was fitted to the projected points. The inner ellipse denotes the region of one standard deviation in both principal directions from the setpoint, the outer ellipse denotes the region of two standard deviations. 
realizable plane. The points of intersection of the ribs of the standard reference cube or their extensions with the realizable plane are arbitrarily numbered (1 through 12) in Fig. 2. These points play a central role in the translation of the definitions of the various disturbances as tabulated in Table 1 into regions in the realizable plane. This is explained in the following section.

\section{Representation of acid-base conditions in the new chart}

In Fig. 2, the three-dimensional configuration of the acid-base variates is displayed. Having determined that in the projection of all realizable combinations of the variates onto the plane spanned by the first two principal components, no significant loss of information occurs, it is of interest to investigate this projection and the way in which the standard definition of disturbances (Table 1) is represented. All conditions are defined as regions in three-dimensional space, separated by the surface planes of the standard reference cube and/or their extensions. It is a trivial excercise to find the intersections of such regions with the realizable plane, once this plane is known. Fig. 3 is a view of the realizable plane, in which these points of intersection are displayed using the same numbering as in Fig. 2. The realizable plane is now subdivided in four types of regions:

- All variates within their respective standard reference ranges. This is the region of standard normals, appearing as the central hexagon.

- One variate outside its standard reference range. These regions appear as triangles sharing one side with the standard normal region. There are six such regions since each of the three variates may be outside its reference range either at the high or at the low end. Triangle $1,5,9$ is such a region; it contains cases in which $\mathrm{pH}, \mathrm{PCO}_{2}$ are within their respective reference range, while $\mathrm{BE}$ is outside at the high end.

- Two variates outside their reference range. These appear as regions, each sharing only one point with the border of the standard normal region. There are six such regions, each touching a different point of the normal hexagon. The region touching in point 1 is such a region; it contains cases for which $\mathrm{pH}$ is within reference; $\mathrm{PCO}_{2}$ and $\mathrm{BE}$ are outside, both at the high end. Therefore, fully compensated respiratory acidoses are represented in this region. (If the patient receives artificial respiration, states of fully compensated metabolic alkalosis are also represented in this region.)

- All three variates are outside their standard reference range. These appear as 'triangular' regions (one side of the triangle is in infinity) with no point in common with the border of the normal reference region. Again, there are six such regions. The region containing point 9 is one of these. It contains cases with all three variates outside their reference range at the high end: partly compensated metabolic alkalosis.

It may be argued that in the representation of Fig. 3, information on the values of the original variates is lost. Indeed, the first two principal axes are linear combinations of the original variates. However, it is still possible to project the original axes onto the realizable plane and without loss of generality this can be done in such a way that the projection of the positive $\mathrm{pH}$ direction points to the right, as explained in Section 3. The projected directions of $\mathrm{pH}, \mathrm{PCO}_{2}$ and $\mathrm{BE}$ are shown in Fig. 3 as lines with arrows and corresponding labels. The arrows originate in the point $\mathrm{pH}=7.40$, $\mathrm{PCO}_{2}=40.00(\mathrm{mmHg}), \mathrm{BE}=0.0$ and point in the direction of increasing value of the corresponding

\section{Table 3}

Means, standard deviations and correlation coeffient of the fit of the transformed distribution as determined from the Gaussian part of 1500 Astrup values

\begin{tabular}{ll}
\hline$x(\mathrm{pH})$ & 1.273 \\
$y$ & 0.247 \\
$\sigma_{x}$ & 2.413 \\
$\sigma_{y}$ & 2.546 \\
$\mathrm{cc}$ & 0.259 \\
\hline
\end{tabular}

The 1500 measurements and the ellipses corresponding to one and two standard deviations are plotted in Fig. 4. 
variate. Therefore, even if these projected directions are no longer perpendicular, each condition as represented by a point in the plane may still be interpreted in terms of an increased or decreased value of each of the original variates, by (mentally) projecting the point on the corresponding arrowed direction. Since the projection is a linear one, a linear scale may be added to the projected variate

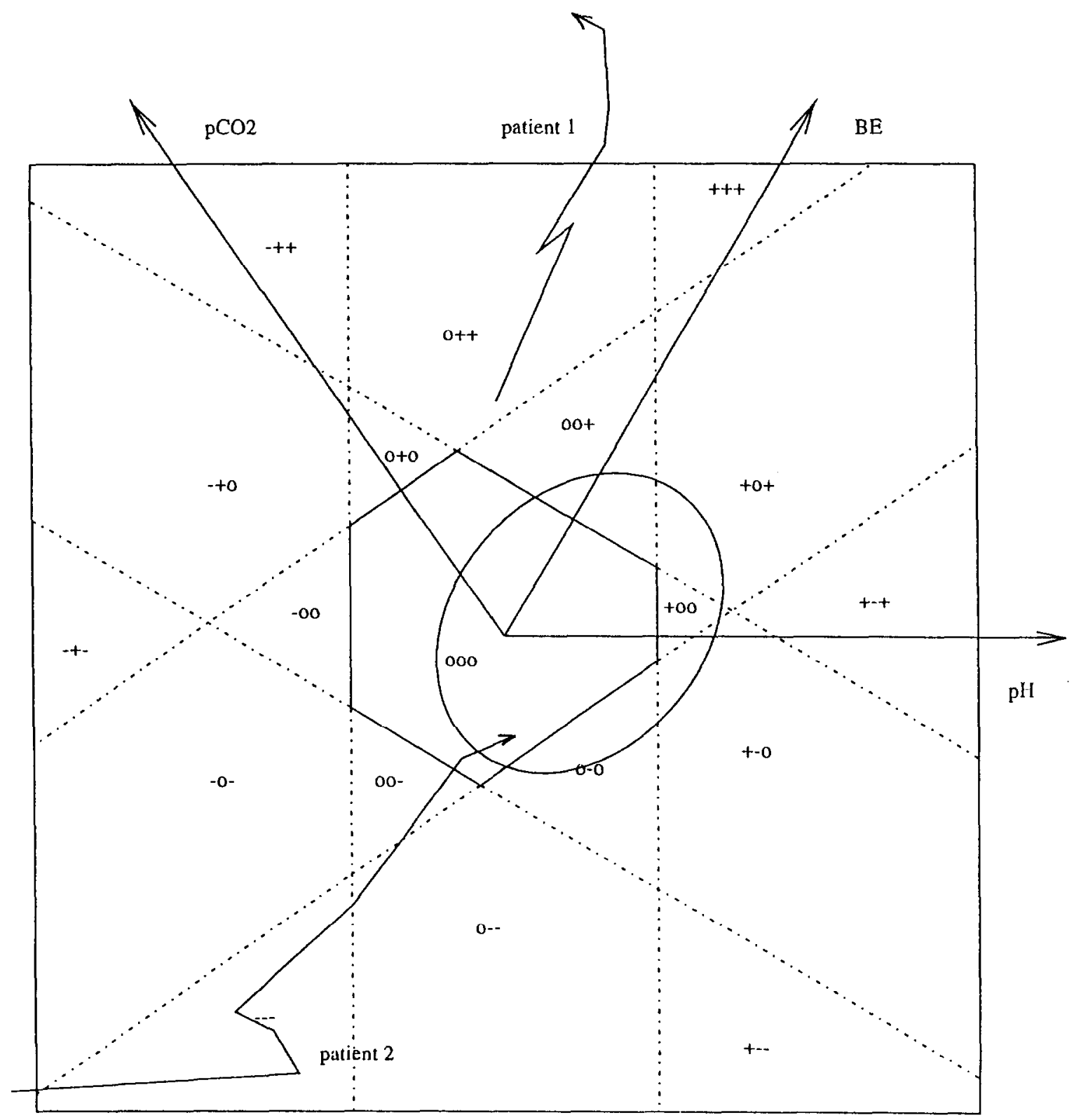

Fig. 5. Two patient trajectories represented in the new acid-base chart. Relevant data of both patients can be found in Table 5. The representation of the trajectories is practically distortionless with respect to the three-dimensional configuration. The arrows in the trajectories point in the direction of time. 


\section{SIGGAARD-ANDERSEN ACID-BASE CHART}

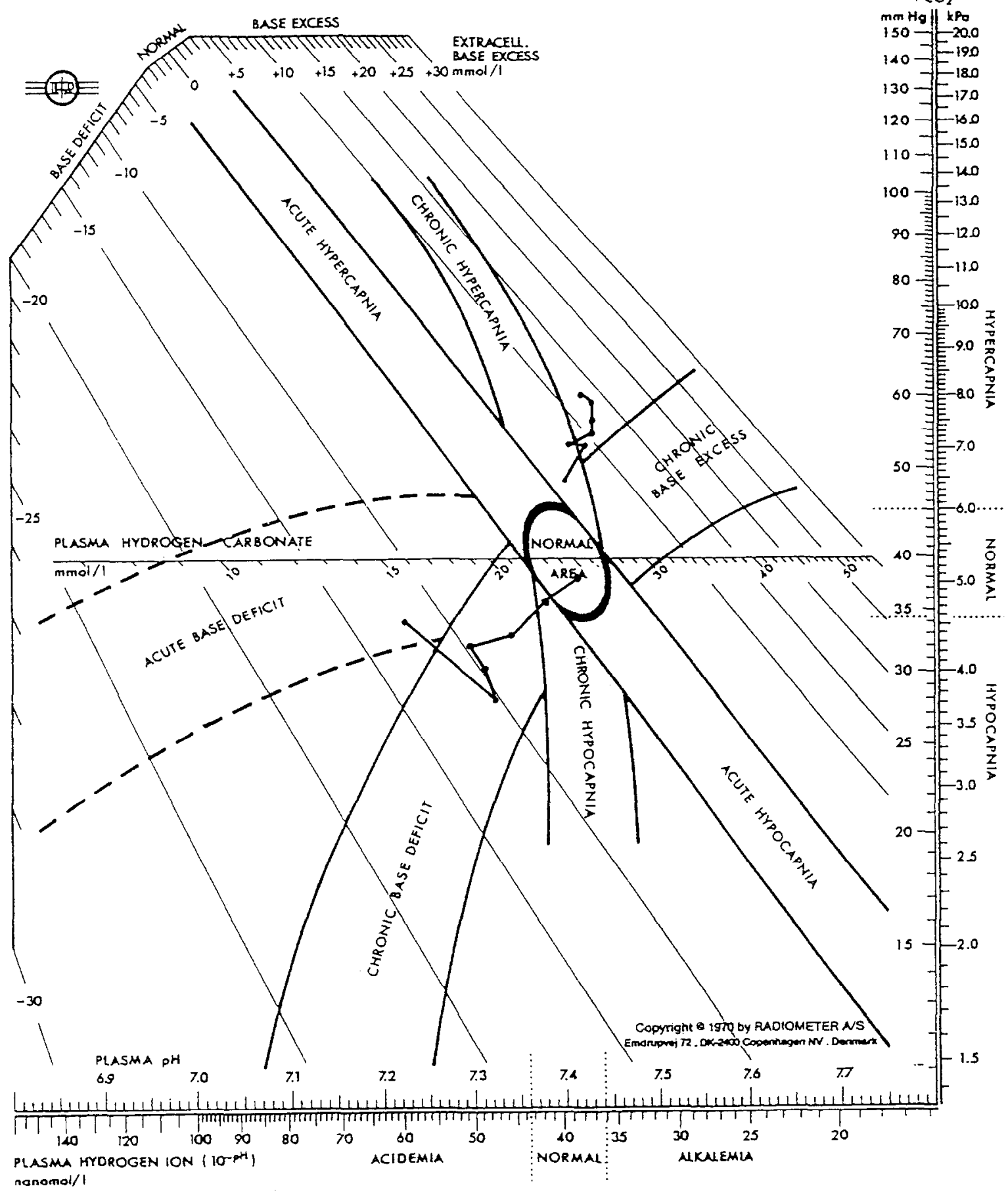

Fig. 6. The same patient trajectories as in Fig. 5. now plotted in the classical Siggaard-Andersen diagram. The distortion of the line in comparison with Fig. 5. is apparent. 
Table 4

Percentage of variance explained by the first two principal components and the direction coefficients of the normal to the plane spanned by the first two principal components for three data sets of 1500 observations each, obtained from three different laboratories

\begin{tabular}{lllll} 
& $\%(1+2)$ & $e_{31}$ & $e_{32}$ & $e_{33}$ \\
\hline Data set 1 & 99.7 & 0.639 & 0.565 & -0.522 \\
Data set 2 & 99.7 & 0.613 & 0.499 & -0.612 \\
Data set 3 & 99.2 & 0.594 & 0.567 & -0.571 \\
\hline
\end{tabular}

directions. A representation similar to the one outlined here has been proposed in the past [11]. Baird Hastings reports: 'I still think that . . . triaxial coordinate paper $\ldots$ is ideal for plotting acid-base paths, but I have never been able to persuade anyone else that this is so, so I've stopped trying to do so'. Although the representation in Ref. 11 is similar, no justification in terms of principal components is given and, therefore, the directions of the axes representing the three variates (at angles of $120^{\circ}$ to each other) remain arbitrary. Fig. 4 is a view of the realizable plane, in which the 1500 points, each corresponding to a patient condition, have been plotted. A procedure as described by Gelsema et al. [2] was used to calculate the characteristics of a Gaussian distribution,

Table 5

Time of blood gas analysis, $\mathrm{pH}, \mathrm{PCO}_{2}$ (in $\mathrm{kPa}, 1 \mathrm{kPa}=7.50$ $\mathrm{mmHg}$ ) and $\mathrm{BE}$ (in $\mathrm{mmol} / \mathrm{l}$ ) of the two patients represented in Figs. 5 and 6.

\begin{tabular}{cccc}
\hline Time of analysis & $\mathrm{pH}$ & $\mathrm{PCO}_{2}$ & $\mathrm{BE}$ \\
\hline Patient l & & & \\
$06: 00 \mathrm{~h}$ & 7.40 & 6.5 & 4.2 \\
$10: 00 \mathrm{~h}$ & 7.42 & 7.1 & 8.6 \\
$12: 00 \mathrm{~h}$ & 7.41 & 7.1 & 7.7 \\
$16: 00 \mathrm{~h}$ & 7.43 & 7.3 & 10.5 \\
$20: 00 \mathrm{~h}$ & 7.43 & 7.5 & 11.3 \\
$22: 00 \mathrm{~h}$ & 7.43 & 7.9 & 12.6 \\
$24: 00 \mathrm{~h}$ & 7.42 & 8.0 & 12.6 \\
Patient 2 & & & \\
$21: 00 \mathrm{~h}$ & 7.22 & 4.5 & -13 \\
$23: 00 \mathrm{~h}$ & 7.32 & 3.7 & -9.8 \\
$01: 00 \mathrm{~h}$ & 7.31 & 4.0 & -9.2 \\
$02: 00 \mathrm{~h}$ & 7.30 & 4.3 & -9.4 \\
$04: 00 \mathrm{~h}$ & 7.34 & 4.4 & -6.2 \\
$06: 00 \mathrm{~h}$ & 7.38 & 4.8 & -2.4 \\
$10: 00 \mathrm{~h}$ & 7.42 & 5.1 & -2.5 \\
\hline
\end{tabular}

disregarding possible outliers. This procedure yielded values as listed in Table 3. The two ellipses corresponding to, respectively, one and two standard deviations in each principal direction of this fitted normal distribution are also indicated.

The mean of the distribution is the setpoint of the clinic and, based on a study of the patient population, an ellipse concentric with those shown in Fig. 4 may be defined to localize those patients who, in the context of the procedures in that clinic. may be regarded as normal. Those conditions with a representation outside the area of normality may then be assigned a disturbance according to the standard classification system, which is readily read off the chart. Alternatively, using the same chart, normality may be defined according to the standard classification, i.e. as the region delimited by the central hexagon. In whichever way normality is defined, conditions represented in one of the triangular areas surrounding the hexagon (undenominables) may be reassigned to the closest denominated area.

Fig. 5 shows two 'patient trajectories' in the new chart. The arrows in the trajectories point in the direction of time. Patient 1 starts just outside the normal area and in a time span of $18 \mathrm{~h}$ moves away from the normal condition, remaining in the area designated to 'completely compensated respiratory acidosis' $(0++)$. The condition of this patient becomes increasingly worse. The trajectory for patient 2 starts on the border of 'uncompensated metabolic acidosis' (-o-) and 'partly compensated metabolic acidosis' (-- ). In a time span of $12 \mathrm{~h}$, the patient moves towards normal, the two last points residing inside the normal area. As a comparison, the two patient trajectories have also been plotted in the classical Siggaard-Andersen diagram, which is a projection on the $\mathrm{pH}-\log \mathrm{PCO}_{2}$ 
plane (Fig. 6). Given the fact that the new chart has a view perpendicular to all trajectories, it is better suited for purposes of patient monitoring. The shortcoming of the classical chart in this respect was already observed in Ref. 4.

\section{Stability of the chart}

In Section 4 it is noted that the transformation leading to the representation of principal components intrinsically depends exclusively on the formula for the calculation of $\mathrm{BE}$ implemented in the equipment used. The determination of the transformation matrix depends, however, on the population used. The transformation as calculated on the basis of two other sets of 1500 Astrups, obtained from two other clinics, using different instruments were compared with the one presented in Section 4. The results are presented in Table 4 in terms of the percentage of the total variance explained by the two first principal components and in terms of the direction coefficients of the third principal component (this is the normal to the plane spanned by the first two principal components). The first parameter is a measure of the faithfulness of the projections to the threedimensional configuration $(100 \%$ implies no distortion). The direction coefficients may be used to calculate the angles between the various realizable planes. It is apparent that in all three data sets, the variance explained by the first two principal components exceeds $99 \%$. The smaller value in Data set 3 may be explained by the fact that in this set, BE was available only to the nearest integer value, whereas in the other sets $\mathrm{BE}$ was expressed to the precision of one decimal place. The cosine of the angle between pairs of planes is always larger than 0.993 . Thus, the three planes almost coincide.

\section{Conclusions}

The principal component transformation is introduced as a technique allowing the representation of acid-base conditions in two dimensions. The transformation depends only on the formula for the calculation of $\mathrm{BE}$ implemented in the equipment used. The standard definitions of the various acid-base disturbances may be easily translated into regions in the plane spanned by the first two principal components. It is therefore possible to design a chart for a particular instrument, with the regions corresponding to the standard definitions drawn into it. The representation of patient data as a function of time as trajectories is for all practical purposes faithful to the threedimensional configuration and could, for example, be presented at the bedside on a display. The distortion, measured as a percentage of variance thus not represented, is shown to be less than $0.7 \%$ for the three different types of instruments investigated.

This latter point may also serve as a plausibility check for Astrup values: each combination of $\mathrm{pH}$, $\mathrm{PCO}_{2}$ and $\mathrm{BE}$ values must lead to an extremely small third principal component. If this is not the case for a given combination, the combination must be regarded as invalid.

\section{Acknowledgements}

The authors are indebted to Dr R.N.M. Weijers and Dr C.P. Stoutenbeek for making a collection of Astrup values from the Onze Lieve Vrouwe Gasthuis in Amsterdam available. Likewise, Dr B. van der Berg (Academic Hospital Dijkzigt, Rotterdam) and Dr R.W. Wulkan (Sophia Children's Hospital, Rotterdam) contributed similar material from their respective clinics.

\section{References}

1 Gelsema ES, Leijnse B and Wulkan RW: A multidimensional analysis of three chemical quantities in the blood, Med Inf 16(1) (1991) 43-54.

2 Gelsema ES, Leijnse B and Wulkan RW: Detection of aberrant observations in a background of an unknown multidimensional Gaussian distribution, Methods Inf Med, 29 (1990) 236-242.

3 Madias NE, Adroqué HJ, Horowitz GL, Cohen JJ and Schwartz WB: A redefinition of normal acid-base equilibrium in man: carbon dioxide tension as a key determinant of normal plasma bicarbonate concentration, Kidney Int, 16 (1979) 612-618.

4 Siggaard Andersen O: An acid-base chart for arterial blood with normal and pathophysiological reference areas, Scan J Clin Lah Invest, 27(3) (1971) 239-245.

5 Astrup P, Jørgensen K, Siggaard-Andersen $O$ and Engel 
K: The acid-base metabolism, a new approach, Lancet, i (1960) 1035-1039

6 Bunker JP: The great trans-Atlantic debate, $J$ Anesthesiol, 26 (1965) 591-594.

7 Schwartz WB and Relman AS: A critique of the parameters used in the evaluation of acid-base disorders. 'Whole-blood buffer base' and 'standard bicarbonate' compared with blood $\mathrm{pH}$ and plasma bicarbonate concentration, N Engl J Med, 268 (1963) 1382-1388.

8 Rose BD: Clinical Physiology of Acid-base and Electrolyte Disorders, third edition, McGraw-Hill, New York, 1989.
9 Van Slyke DD: Some points of acid-base history in physiology and medicine. In: Current Concepts of Acid-base Measurement (Ed: H.E. Whipple), The New York Academy of Sciences, New York, 1964, pp. 5-13.

10 Siggaard-Andersen $O$ : Titratable acid or base of body fluids. In: Current Concepts of Acid-base Measurement (Ed: H.E. Whipple), The New York Academy of Sciences, New York, 1964, pp. 41-57.

11 Baird Hastings A: Acid-base measurements in vitro. Introductory remarks. In: Current Concepts of Acid-base Measurement (Ed: H.E. Whipple), The New York Academy of Sciences, New York, 1964, pp. 15-24. 